\title{
Inflammation, atrophy, and gastric cancer
}

\author{
James G. Fox' and Timothy C. Wang ${ }^{2}$ \\ ${ }^{1}$ Division of Comparative Medicine, Massachusetts Institute of Technology, Cambridge, Massachusetts, USA. ${ }^{2}$ Division of Digestive and Liver Diseases, \\ Columbia University Medical Center, New York, New York, USA.
}

\begin{abstract}
The association between chronic inflammation and cancer is now well established. This association has recently received renewed interest with the recognition that microbial pathogens can be responsible for the chronic inflammation observed in many cancers, particularly those originating in the gastrointestinal system. A prime example is Helicobacter pylori, which infects $\mathbf{5 0 \%}$ of the world's population and is now known to be responsible for inducing chronic gastric inflammation that progresses to atrophy, metaplasia, dysplasia, and gastric cancer. This Review provides an overview of recent progress in elucidating the bacterial properties responsible for colonization of the stomach, persistence in the stomach, and triggering of inflammation, as well as the host factors that have a role in determining whether gastritis progresses to gastric cancer. We also discuss how the increased understanding of the relationship between inflammation and gastric cancer still leaves many questions unanswered regarding recommendations for prevention and treatment.
\end{abstract}

\section{The incidence and classification of gastric cancer}

Although gastric cancer was the leading cause of death from cancer in the United States and Western Europe as recently as 1930, with incidence rates in males exceeding 45 per 100,000, a decline in both incidence and mortality in every country where cancer mortality figures are recorded has been noted (1). Despite this international trend, gastric cancer remains the fourth most common cancer and the second leading cause of cancer-related death worldwide (2).

Gastric tumors are classified anatomically as proximal (also known as cardia) and distal (also known as noncardia); the latter are commonly associated with infection with Helicobacter pylori, but the former are not (Figure 1). Gastric adenocarcinomas are defined as well differentiated (also known as intestinal-type) and undifferentiated (also known as diffuse-type) (3). Intestinal-type tumors are characterized by a corpus-dominated gastritis with gastric atrophy and intestinal metaplasia, whereas diffuse-type tumors are characterized by gastritis throughout the stomach but no atrophy (4). Intestinal-type tumors are found predominantly in geographic areas with a high incidence of gastric cancer, whereas diffuse-type tumors are found more uniformly throughout the world.

\section{Atrophic gastritis and gastric cancer}

Although a number of factors probably influence an individual's predisposition to gastric cancer and course of progression to gastric cancer, it is clear that chronic inflammation is a feature that links this cancer to many other types of malignancy (5). Studies, conducted in the first half of the 20th century, of surgical resection specimens suggested that chronic gastritis was more advanced in individuals with gastric cancer than in individuals with duodenal ulceration $(6,7)$. Investigators also recognized that areas of gastric adenocarcinoma were frequently found in areas of chronic inflammation, as well as in settings of atrophic gastritis. Earlier literature, based on studies in several countries, indicated that by the fifth decade of life, more than half the individuals

Nonstandard abbreviations used: BMDC, bone marrow-derived cell; Le ${ }^{\mathrm{b}}$, Lewis $\mathrm{B}$; NOD1, nucleotide-binding oligomerization domain-1; PAI, pathogenicity island; PPI, proton-pump inhibitor; SHH, sonic hedgehog; SPEM, spasmolytic polypeptideexpressing metaplasia; Vac, vacuolating cytotoxin.

Conflict of interest: The authors have declared that no conflict of interest exists. Citation for this article: J. Clin. Invest. 117:60-69 (2007). doi:10.1172/JCI30111. sampled on a random basis had gastritis (8). Correa recognized that this form of gastritis was multifocal, appearing in all areas of the stomach, and was most prevalent in the same population that had an increased risk of developing gastric cancer $(9,10)$. He also observed that populations at high risk of developing gastric cancer in Colombia had a higher prevalence of atrophic gastritis than populations at low risk of developing gastric cancer (11), substantiating observations made by others $(12,13)$. It is now known that H. pylori, which infects $50 \%$ of the world's population, is a major factor in both the induction of atrophic gastritis and histological progression to gastric cancer.

Less is known about the histological changes that occur during the progression to diffuse-type gastric cancer than about those that occur during the progression to intestinal-type gastric cancer, which evolves through a series of discrete steps known as the Correa pathway $(14,15)$ (Figure 2). Although gastric atrophy and intestinal metaplasia are distinct steps in the pathway, these terms have sometimes been used synonymously. However, most gastric pathologists define atrophic gastritis as the loss of specialized glandular tissue, for example, loss of the oxyntic glands (which contain parietal cells) in the gastric corpus (16). By contrast, intestinal metaplasia is defined as replacement of original gastric glands with straight tubular crypts lined by alternating absorptive and goblet cells and accompanied by inflammatory infiltrates in the lamina propria (11). The development of gastric atrophy was recognized as a critical step in the Correa pathway to intestinal-type gastric cancer, and accumulating evidence indicates that gastric atrophy is much more consistently associated with gastric cancer than is intestinal metaplasia. Gastric atrophy therefore appears to be a better indicator of gastric cancer risk than is intestinal metaplasia. Detailed mapping studies of resected stomachs from patients with intestinal-type gastric cancer have shown that atrophic gastritis, but not intestinal metaplasia, is present in every case (17). Atrophy is generally present as either a multifocal or a diffuse pattern in gastric tissue and is, by definition, associated with the presence of a form of mucous metaplasia that has been termed pseudopyloric metaplasia, also known as spasmolytic polypeptide-expressing metaplasia (SPEM). SPEM is more strongly associated with gastric cancer than is intestinal metaplasia and might be the precursor to the cancerous lesion $(18,19)$. In some mouse models of gastric cancer, SPEM consistently emerges in the 


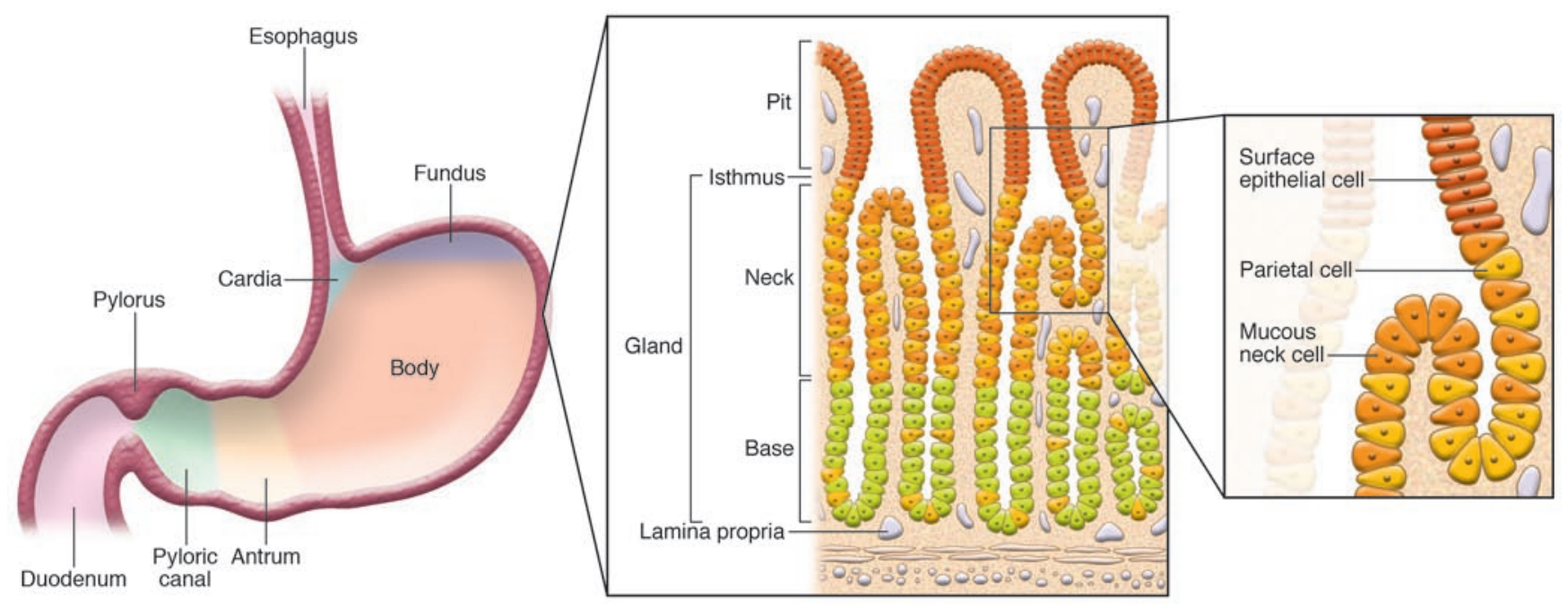

Figure 1

Diagram depicting anatomy of the stomach and histological representation of the oxyntic glands of the body of the stomach. It is these glands, which include parietal cells, that are lost in gastric atrophy. Adapted with permission from W.B. Saunders Co. (122).

chronically inflamed stomach and clearly precedes and gives rise to gastric cancer $(20,21)$.

One possible explanation linking parietal cell loss during gastric atrophy and progression to metaplasia is that the loss of parietal cells is associated with a reduction in the amount of numerous secreted signals that modulate the growth and differentiation of gastric progenitors. This could result in an increase in proliferation and accumulation of undifferentiated progenitors, as seen when parietal cells are specifically ablated in transgenic mice (22). Sonic hedgehog $(\mathrm{SHH})$, a member of the family of Hedgehog proteins known to exert important regulatory functions in patterning and growth in a large number of tissues during embryogenesis, might be one of the secreted signals lost during progression to atrophy. Immunohistochemical studies in both mice and humans indicate that $\mathrm{SHH}$ is expressed by parietal cells (23), and patients with atrophic gastritis exhibit lower levels of SHH immunostaining (24). Importantly, SHH-deficient mice develop a metaplasia of the stomach (25). Because parietal cells are important in regulating complex programs of cellular growth and differentiation in the gastric mucosa, it is not surprising that loss of parietal cells leads to the emergence of metaplastic lineages. Indeed, acute, drug-induced ablation of parietal cells leads to the rapid and reversible development of SPEM $(26,27)$. Studies have pointed to a combination of parietal cell loss and chronic inflammation as necessary for further progression along the metaplasia-dysplasia-cancer pathway (28).

\section{Role of bacterial virulence factors in $\mathrm{H}$. pylori persistence and gastric cancer}

The natural course of infection with $H$. pylori is extremely variable. Most infected individuals remain asymptomatic, and infection with $H$. pylori is not sufficient to induce gastric cancer. Therefore, other factors - known bacterial and host cofactors, and perhaps unknown cofactors - are required. Infection is typically acquired early in life and is followed by a long quiescent phase when there is a chronic gastritis of variable intensity but with minimal symptoms. Peptic ulcer disease tends to develop in patients in their 20 s and 30s, whereas gastric cancer arises several decades later. Only
$10 \%-15 \%$ of individuals infected with $H$. pylori develop peptic ulcer disease, and the risk of gastric cancer is estimated to be approximately 1\%-3\% (29). Interestingly, patients who develop H. pyloriassociated duodenal ulcers seem to be somewhat protected from developing gastric cancer (30). The mechanisms of protection from gastric cancer development include a higher basal level of acid secretion, which in some instances could be related to a genotype that results in low levels of expression of IL-1 $\beta$. The location and severity of gastritis also determine the risk of progression to gastric cancer. Antral gastritis represents a lower cancer risk than corpus gastritis, which affects the body and proximal portions of the stomach. Therefore, the outcomes of infection with $H$. pylori - progression to atrophy and cancer, duodenal ulcers, and asymptomatic status - seem to represent relatively distinct pathways resulting in distinct clinical and pathological outcomes and are probably determined by both bacterial and host factors.

The bacterial factors that enable $H$. pylori to persist in the gastric lumen are still being studied, but considerable insight has been gained through the analysis of the $H$. pylori genome, the creation of $H$. pylori isogenic mutants, and the use of animal models. Critical factors allowing $H$. pylori colonization and survival in the host include the ability of the bacteria to produce large amounts of urease, which hydrolyzes urea to ammonia and carbon dioxide and thereby has a role in neutralizing gastric acid; the presence of flagella, which allow the bacteria to be motile, navigate, and persistently colonize the mucus layer overlying the gastric epithelium; and the ability of the bacteria to produce enzymes that break down the surfactant layer over the gastric epithelium, which allows the bacteria access to nutritional components of the host.

Most of the bacteria exist in a nonadherent, extracellular, mucous environment, which might account for the inefficacy of immune-mediated clearance and the difficulty in achieving antibiotic-mediated eradication. Although about $10 \%$ of $H$. pylori can adhere to gastric epithelial cells, organisms are rarely found intracellularly (31-33). Adherence of $H$. pylori to the gastric epithelium is a complicated process that involves a number of bacterial cell-surface receptors, including the Lewis B-bind- 




ing (Le ${ }^{\mathrm{b}}$-binding) adhesin BabA (34), an outer membrane protein encoded by $b a b A 2$ that binds the histo-blood group antigen $\mathrm{Le}^{\mathrm{b}}$ on the surface of epithelial cells. Even $H$. pylori strains that do not bind Le ${ }^{\mathrm{b}}$ usually possess silent $b a b \mathrm{~A}$ gene sequences that can be activated by recombination events (35). Infection with $H$. pylori strains containing $b a b A 2, \operatorname{cagA}$, and the vacuolating cytotoxin (Vac) allele vacAS1 confers the highest risk of gastric cancer (36). Lewis $\mathrm{X}$, a glycosphingolipid, is an established tumor antigen and biomarker for gastric dysplasia (37). The H. pylori protein SabA (sialic acid-binding adhesin) binds Lewis $\mathrm{X}$, and this interaction also contributes to $H$. pylori adherence, which allows close interactions with the gastric epithelium (38). The distribution of Lewis X mirrors the expression of trefoil factor 1 , which also serves as a binding factor for $H$. pylori, as does $\operatorname{Muc5AC}(39,40)$.

The cag locus. The 2 genetic loci linked with virulence and inflammatory responses that have been most studied are the cag locus and the vacA gene (41). Infection with $H$. pylori strains that contain the cag locus, a 40-kb region of DNA known as the cag pathogenicity island (cag PAI), confers a higher risk for gastric cancer than infection with strains that do not $(42,43)$. The association between the cag PAI and gastric cancer is particularly manifest for intestinal-type gastric cancer. Indeed, although diffuse-type gastric cancer is strongly associated with infection with $H$. pylori, it is equally associated with strains containing the cag PAI and those lacking it (42). Individuals infected with $H$. pylori strains containing the cag PAI have also been shown to have an increased likelihood of having mutations in the gene encoding p53 (44). This correlates with the observation that $p 53^{+/-}$mice transgenic for the lacI gene (which enables researchers to measure the rate of genetic

\section{Figure 2}

Proposed Correa pathway of pathological events in gastric adenocarcinoma. In well-differentiated, intestinal-type gastric cancer, histopathological studies indicated that chronic $H$. pylori infection progresses over decades through stages of chronic gastritis, atrophy, intestinal metaplasia, dysplasia, and cancer (123). The development of cancer has been attributed to alterations in DNA caused by chronic inflammation, recruitment and engraftment of bone marrow-derived cells, an imbalance between epithelial cell proliferation and apoptosis, and, in a milieu of atrophy and achlorhydria, gastric colonization by enteric bacteria with nitrate reductase activity, which facilitates the formation of carcinogenic nitrosamines. Corpus-predominant atrophy, or the loss of specialized glandular cell types such as parietal and chief cells, appears to be the critical initiating step in the progression toward cancer. Adapted with permission from the New England Journal of Medicine (14).

mutations) that are chronically infected with Helicobacter felis have a cumulative increase in gastric tissue mutations and ostensibly an increased cancer risk (45).

A functional cag PAI encodes a type IV secretion system that translocates CagA (which is also encoded by the cag PAI) into gastric epithelial cells (Figure 3). Once internalized, tyrosine residues of CagA are phosphorylated by SRC family kinases, and phosphorylated CagA then activates SRC-homology 2 domain-containing protein tyrosine phosphatase 2 (SHP2) and ERK. This process results in morphological derangement of epithelial cells (46, 47). On the basis of differences in their tyrosine phosphorylation motifs (TPMs), researchers have suggested that distinct CagA proteins have different biological activities (48). These differences in the TPMs of CagA proteins from strains of $H$. pylori isolated in different parts of the world might explain, in part, the marked differences in gastric cancer noted in these different geographic regions.

Strains of $H$. pylori containing a functional cag PAI can induce epithelial cells to produce proinflammatory modulators such as IL-8 in vitro (49). Isogenic mutants lacking 1 or several genes of the cag PAI do not have these in vitro effects, whereas $H$. pylori isogenic mutants lacking $\operatorname{cag} A, \operatorname{cag} F$, or $\operatorname{cag} N$ are still able to induce IL-8 production in vitro $(41,50,51)$.

It was noted that epithelial cells sense $H$. pylori through nucleotide-binding oligomerization domain-1 (NOD1), an intracellular pathogen-recognition molecule with specificity for peptidoglycan of Gram-negative bacteria (52). NOD1 detection of $H$. pylori depended on the delivery of peptidoglycan into host cells by the H. pylori type IV secretion system encoded by the cag PAI (52). It seems that NOD1 represents a key molecule in innate immune sensing of strains of $H$. pylori containing the cag PAI by epithelial cells (Figure 3).

vacA alleles. VacA is a secreted protein that has a vacuolating effect in several cell lines, including gastric cell lines (53). Isogenic mutants lacking the toxin do not induce a vacuolating effect in cell lines $(54,55)$. There is considerable genetic diversity among the vac alleles. The most extensively studied has been the $\mathrm{s} 1 / \mathrm{m} 1$ vacA allele, which typically encodes VacA proteins with a high level of vacuolating activity in vitro (56). The $\mathrm{s} 1 / \mathrm{m} 1 \mathrm{vacA}$ allele has also been associated with augmented inflammation and consistently linked to a higher risk of developing cancer (57-60). Recent data, with direct relevance to the role of VacA in pathogenesis and the persistence of $H$. pylori infection, indicate that VacA efficiently blocks proliferation of $\mathrm{T}$ cells by inducing cell cycle arrest in the 


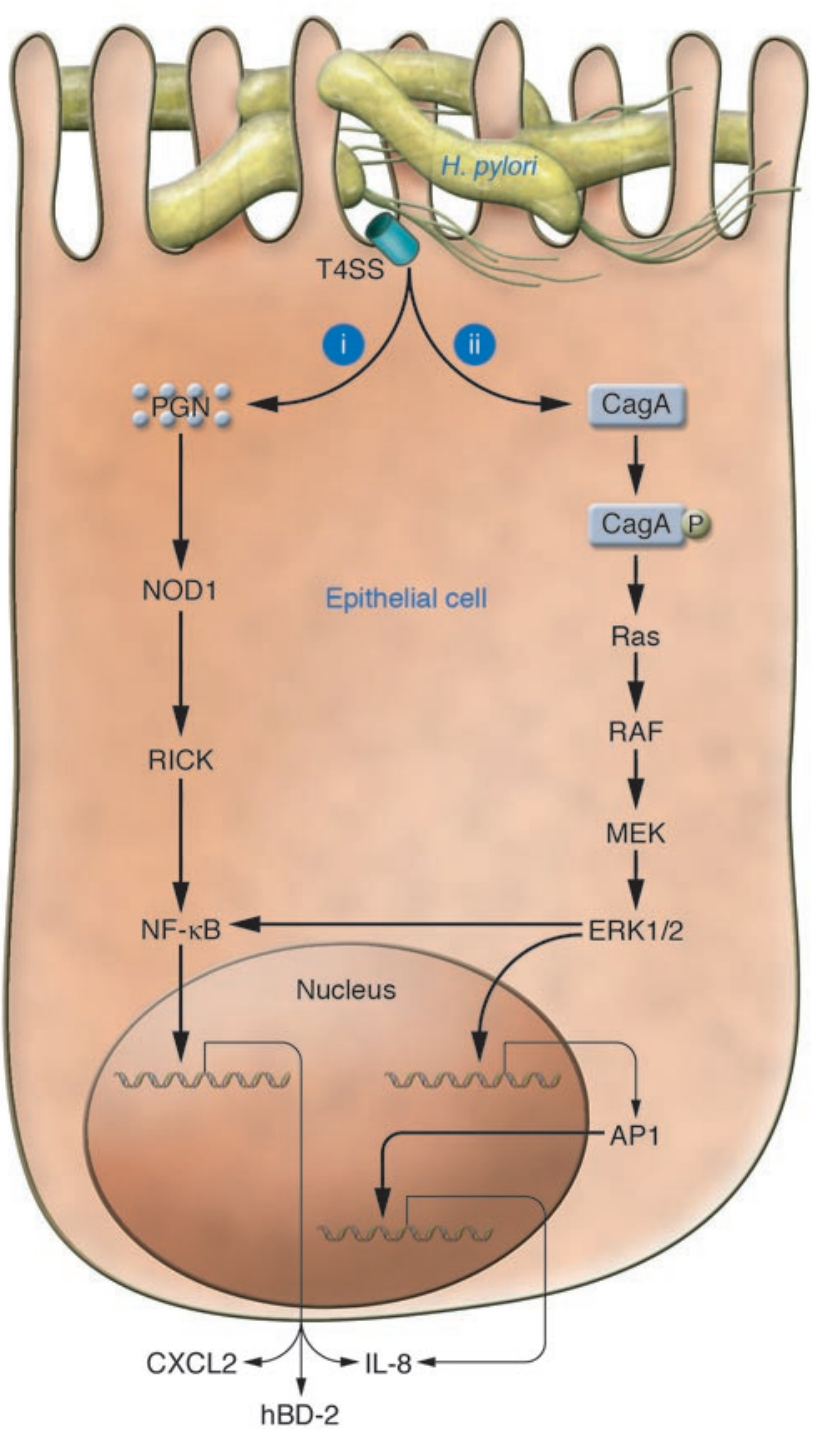

$\mathrm{G}_{1} / \mathrm{S}$ phase (61). VacA might also interfere with signaling downstream of the TCR and IL-2 receptor. These changes could help explain the persistence of $H$. pylori infections (61).

Overall, bacterial factors clearly have a role in the induction of both inflammation and epithelial cell injury, and strains of $H$. pylori that contain a functional cag PAI are more strongly associated with a risk of developing cancer. Nevertheless, specific bacterial factors have not been shown to have a direct role in the loss of parietal cells and chief cells, which leads to gastric atrophy.

\section{Host factors that affect the development of gastric cancer}

Genetic evidence of a role for immunity. Although bacterial factors have an important role in disease pathogenesis, most evidence suggests that host factors are paramount in determining progression to gastric cancer. The risk of developing gastric cancer is increased up to 3 -fold in individuals with an immediate blood relative with gastric cancer, and $10 \%$ of cases show familial clustering (62). Infection with $H$. pylori is likely to occur throughout a family, and thus part of this familial occurrence could be related to intrafamilial transmission of $H$. pylori. However, even when $H$. pylori infection is

\section{Figure 3}

H. pylori induces proinflammatory responses in epithelial cells by 2 pathways. H. pylori-induced proinflammatory responses are dependent on the presence in $\mathrm{H}$. pylori of a functional type IV secretion system (T4SS), which delivers effector molecules, such as cell wall peptidoglycan (PGN) and the protein CagA, to epithelial cells. The precise mechanisms by which the $H$. pylori T4SS mediates effector delivery to host cells are, however, presently unknown. (i) Recognition of $H$. pylori PGN by the cytosolic host defense molecule NOD1 leads to NF-кB activation. On the basis of studies with Shigella flexneri, it is likely that NOD1 activates NF-kB via caspase-recruitment domain (CARD)-CARD interactions with receptor-interacting serine-threonine kinase (RICK; also known as RIP2). Activated NF-кB complexes translocate to the nucleus, where they upregulate expression of genes encoding the proinflammatory chemokines IL- 8 and CXC-chemokine ligand 2 (CXCL2; also known as MIP2) and the antimicrobial peptide human $\beta$-defensin-2 (hBD-2). (ii) CagA translocation into epithelial cells by certain $H$. pylori strains able to induce high levels of IL-8 is also accompanied by the induction of an inflammatory response. Tyrosine phosphorylation of EPIYA motifs on CagA triggers a signaling cascade that involves the Ras-dependent kinases ERK1 and ERK2, leading to activation of the transcription factors NF-KB and activator protein-1 (AP-1) and, ultimately, IL-8 production by host cells. Courtesy of R. Ferrero (Monash University, Clayton, Victoria, Australia).

controlled for, a family history of gastric cancer remains a risk factor for developing the disease, suggesting that there is indeed an important genetic component to this. Nevertheless, only a small part of the familial clustering of gastric cancer is attributable to known family cancer syndromes. Instead, the observation that relatives of patients with gastric cancer had a higher prevalence of atrophy and hypochlorhydria suggested genetic predisposition to the development of atrophy, the precursor of gastric cancer. This increased prevalence of atrophy was limited only to those family members who were also infected with $H$. pylori (63), leading to the hypothesis that genetic factors cause a more intense immune response to $H$. pylori and that this results in atrophic gastritis.

The search for genetic factors that predispose to gastric cancer has also capitalized on the observation that patients who progress to atrophy and cancer secrete lower levels of gastric acid compared with patients with duodenal ulcers. Thus, the initial genetic study of families with an increased incidence of precancerous changes focused on IL-1 $\beta$, a well-known proinflammatory cytokine that is also a powerful inhibitor of acid secretion in the stomach. The $I L-1 \beta$ gene cluster includes both $I L-1 \beta$ and $I L-1 R N$, the gene that encodes the naturally occurring IL-1 receptor antagonist. A study of white subjects from Poland and Scotland demonstrated that H. pylori-infected individuals with either $I L-1 \beta-31{ }^{*} C$ or $I L-1 \beta-511 * T$ together with $I L-1 R N^{*} 2 / I L-1 R N^{*} 2$ had a 2 - to 3 -fold increased risk of developing gastric atrophy and gastric cancer (63). Portuguese and Japanese investigators also showed a strong association between proinflammatory polymorphisms in the $I L-1 \beta$ gene cluster and an increased risk of developing gastric cancer $(64,65)$. An increased risk for gastric cancer associated with proinflammatory $I L-1 \beta$ polymorphisms has now been confirmed in many populations throughout the world $(60,65,66)$. Later studies also determined genetic polymorphisms in TNF- $\alpha$ and $I L-10$, which, when combined with proinflammatory $I L-1 \beta$ gene cluster polymorphisms, result in a high-risk genotype with a 27 -fold or greater risk of developing gastric cancer (67). Studies have also identified the $-251 T$ allele in the $I L-8$ promoter as significantly associated 


\section{Figure 4}

Histological progression of Helicobacter-induced gastric cancer in a mouse model. Normal: Histology of the body of the stomach. Acute gastritis: Infiltration of mucosal and submucosal lymphocytes with pockets of polymorphonuclear cells, accompanied by mild mucosal defects and edema. Chronic gastritis: Moderate to severe inflammation with marked epithelial defects including gland dilatation and mineralization. Atrophic gastritis: Chronic inflammation with focal fibrosis and complete loss of oxyntic parietal and chief cells. Intestinal metaplasia: Gastric epithelial metaplasia to an intestinal phenotype characterized by columnar elongation, mucous droplets occasionally forming goblet cells, and production of mixed acidic (blue, intestinal-type) and neutral (red, gastric-type) mucins as shown by pH 2.5 Alcian blue/PAS stain (inset). Dysplasia: High-grade glandular dysplasia characterized by irregular size and shape, infolding, branching and cell piling, and marked cellular and nuclear atypia. Cancer: Gastric intraepithelial neoplasia, here with intramucosal invasion (arrow), develops in H. pylori-infected wild-type B6129 mice, as well as in certain genetically engineered models (124-126). Scale bars: $160 \mu \mathrm{m}$ (first panel); $400 \mu \mathrm{m}$ (second through fourth panels); $80 \mu \mathrm{m}$ (fifth panel; inset, original magnification, $\times 400$ ); $40 \mu \mathrm{m}$ (sixth panel); $800 \mu \mathrm{m}$ (seventh panel). Second panel (acute gastritis): adapted with permission from American Journal of Physiology-Gastrointestinal and Liver Physiology (124). All other panels: adapted from Cancer Research $(125,126)$.

with an increased risk of gastric cancer $(68,69)$. These observations provide strong evidence for host genetics in determining progression to gastric cancer and strengthen the connection between the inflammatory response and the development of gastric atrophy.

Experimental evidence of a role for immunity. The importance of chronic inflammation in the pathogenesis of gastric atrophy and gastric cancer has been shown most elegantly using mouse models of infection with Helicobacter spp. (Figure 4). Initial studies revealed that host $\mathrm{T}$ cell immunity was critical for the development of gastric disease in response to infection with Helicobacter spp. (70, 71). Although C57BL/6 mice infected with $H$. felis recapitulated the loss of parietal cells and chief cells observed in human patients, mice lacking $T$ and $B$ cells infected with $H$. felis showed no changes in gastric epithelial cells $(20,21,70,72)$. By contrast, infection of mice lacking $\mathrm{B}$ cells but able to mount a normal $\mathrm{T}$ cell response did lead to severe atrophy and metaplasia. Direct experimental evidence that Th1 cell immune responses are responsible for the progression of gastric preneoplastic lesions has been provided by several studies. First, gastritis is enhanced in $H$. felis-infected immunodeficient mice after transplantation with splenocytes, $\mathrm{CD}^{+} \mathrm{T}$ cells, or Th1 cells from wild-type mice $(72,73)$. Second, mouse strains susceptible to gastric atrophy, such as C57BL/6 mice, mount strong Th1 cell responses after infection with Helicobacter spp. (74), whereas mouse strains resistant to gastric atrophy, such as BALB/c mice, mount Th2 cell responses. Third, deletion of the gene encoding the Th1 cytokine IFN- $\gamma$ protects mice from gastric atrophy induced by infection with Helicobacter spp., whereas deletion of the gene encoding the Th2 cytokine IL-4 leads to more severe atrophic gastritis (75). Finally, infusion of IFN- $\gamma$ into C57BL/6 mice can induce metaplasia and SPEM (76), whereas pretreatment of mice with IL-4 prevents the development of atrophic gastritis after infection with Helicobacter spp. (77).

A recent study has also demonstrated that coinfection of C57BL/6 mice with the parasite Heligmosomoides polygyrus and $H$. felis can polarize the Th1 cell immune response normally induced by infection with $H$. felis toward a Th2 cell response, thereby decreasing the development of gastric atrophy (74). Studies supporting

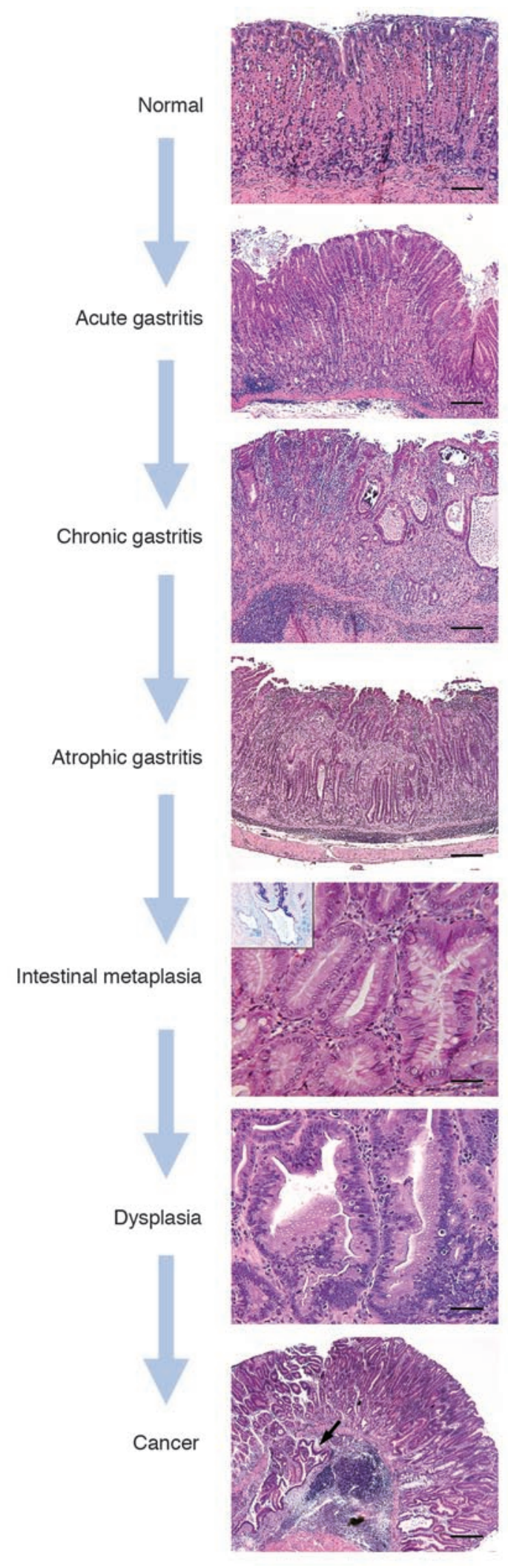

this Th1/Th2 paradigm as the best explanation for geographic variation in gastric cancer rates among humans and the so-called "African enigma" (whereby in Africa rates of infection with H. pylori are high but rates of gastric cancer are low) have used measurement 


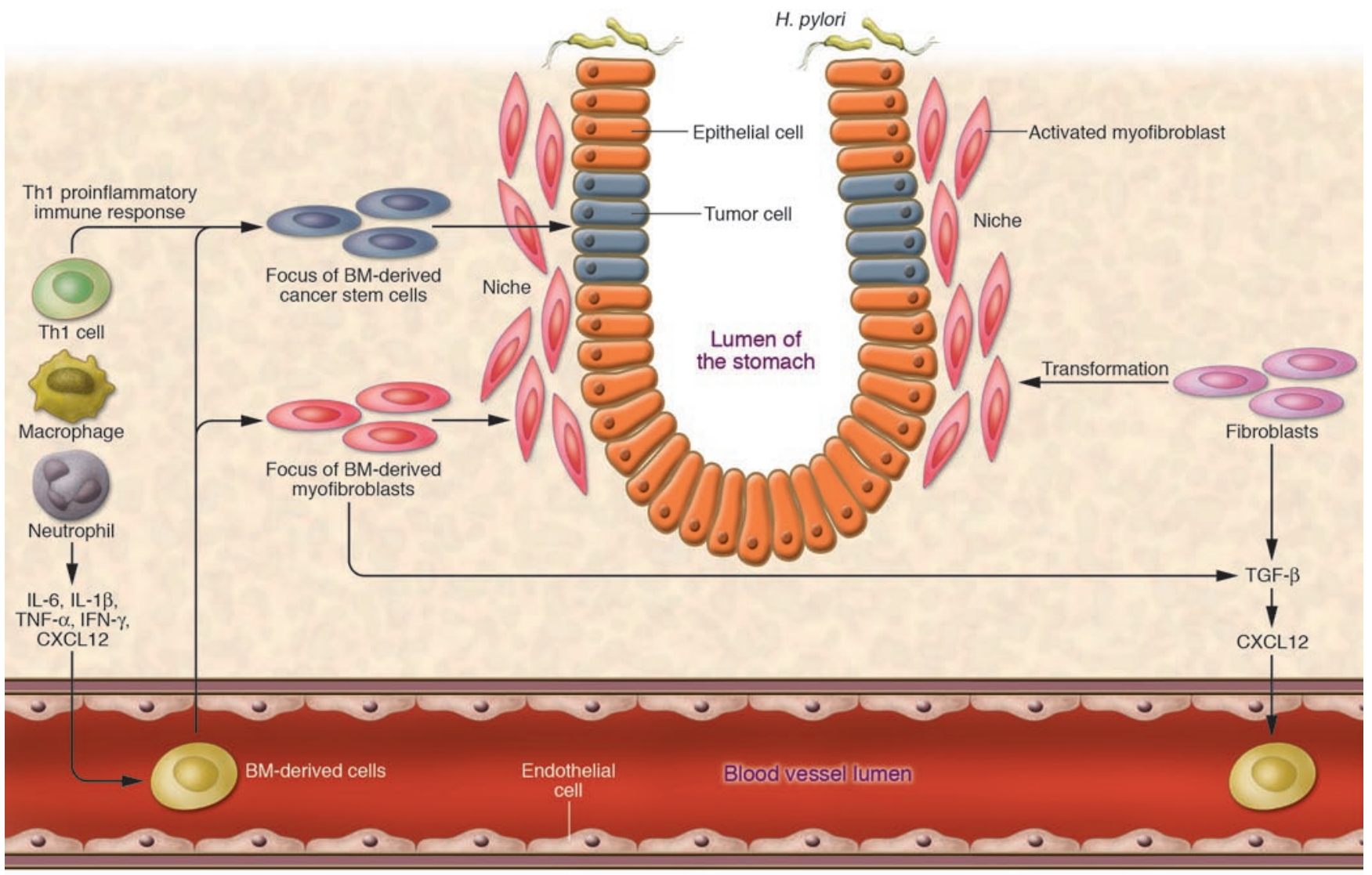

Figure 5

BMDCs in gastric tumor initiation and progression. H. pylori-associated chronic inflammation, driven by macrophages and Th1-polarized lymphocytes, results in increased cytokine and chemokine production that mobilizes and recruits BMDCs from the circulation. The proinflammatory milieu leads to an altered stem cell niche, characterized by increased numbers of activated myofibroblasts, which can originate from both BMDCs and local stromal populations. Some of the BMDCs may also be recruited into the progenitor zone, giving rising to epithelial metaplasia and dysplasia (i.e., cancer stem cells).

of IgG subclass responses as surrogate markers for polarization of Th cell function (78). These studies suggested that adults in undeveloped areas of the world mount predominantly Th2 cell-associated IgG1 responses to infection with $H$. pylori, whereas adults in developed countries mount predominantly Th1 cell-associated IgG2 responses (78); this bias to Th2 cell responses correlates with a higher rate of intestinal helminthiasis (79). Furthermore, individuals infected with $H$. pylori and Schistosoma mansoni have been found to have reduced numbers of apoptotic gastric epithelial cells and decreased gastric epithelial cell proliferation as compared with persons infected with just $H$. pylori (80). Individuals infected with H. pylori and Schistosoma japonicum also had a substantially higher pepsinogen I/II ratio (a surrogate biomarker for gastric atrophy), indicating that the parasitic infection had a protective effect on progression to atrophy (81). Although other factors, such as life expectancy, diet, limited cancer registries, and limited diagnostic capabilities, are probably important, parasitic coinfection is likely to be strongly protective against gastric cancer development.

\section{Chronic inflammation and the recruitment of bone marrow-derived cells}

Chronic inflammation results in the upregulation of diverse cytokines and chemokines and the recruitment of numerous hematopoietic populations to inflamed tissues. Although studies have focused on well-defined immune cell types, recently it has been recognized that chronic inflammation - in addition to inducing the loss of parietal cells and chief cells in the glandular gastric mucosa - stimulates the recruitment of progenitor cell populations to the stomach and their subsequent engraftment. Numerous reports have demonstrated that chronic inflammation leads to the recruitment of bone marrow-derived endothelial progenitors and bone marrow-derived myofibroblasts in mouse models of colitis and hepatitis (82-84). Similar cell populations are recruited to the stomach in Helicobacter-induced gastritis (T.C. Wang, unpublished observations), but studies in this model of gastric cancer have also revealed that chronic inflammation leads to the recruitment of bone marrow-derived epithelial cells (28). Accumulating data support the notion that adult bone marrow-derived cells (BMDCs) have a surprising degree of plasticity, contradicting the conventional view that bone marrow stem cells give rise only to elements in the peripheral blood and marrow stroma (85). Indeed, we showed that in C57BL/6 mice transplanted with marked bone marrow and infected with $H$. felis, most metaplasia, dysplasia, and cancer was donor-derived and therefore arose from BMDCs (28). These initial studies did not support a role for cell fusion in this model, although data from other laboratories have suggested that 
cell fusion is the principal mechanism responsible for bone marrow-derived hepatocytes $(86)$ and intestinal cells $(87,88)$. Interestingly, recruitment of BMDCs to the gastric mucosa was not observed during acute gastric infection with Helicobacter spp., nor was it observed during either acute ulceration or drug-induced parietal cell loss. Instead, BMDC-derived metaplasia arose only in the setting of severe, chronic inflammation (28) (Figure 5).

There is currently considerable interest in defining the association between chronic inflammation and recruitment of BMDCs, and the mechanisms involved. It would be expected to involve upregulation of proinflammatory cytokines (for example, IL-1 $\beta$, TNF- $\alpha$, and IL-6) as well as chemokines, such as CXC-chemokine ligand 12 (CXCL12; also known as SDF), known to contribute to the recruitment of progenitors. Recent studies have pointed to activated myofibroblasts, which are $\alpha$-SMA-expressing stromal cells that make up the stem cell niche, as the probable source of the CXCL12 that mediates progenitor recruitment (89).

\section{Achlorhydria and bacterial overgrowth}

The loss of parietal cells not only leads to a reduction in important differentiation signals but also leads to loss of gastric acid secretion and achlorhydria. The achlorhydric stomach must be considered an abnormal environment, with the potential for bacterial overgrowth. Very few species of bacteria other than Helicobacter spp. can be cultured from either the gastric juice or the gastric mucosal surface of individuals secreting normal levels of gastric acid. However, a range of bacterial species can be cultured from the gastric juice if the intragastric $\mathrm{pH}$ rises above 4.0 (90). These bacteria might replicate and promote many of the pathophysiological changes associated with $H$. pylori infection. Indeed, it is known that colonization with $H$. pylori declines over time in patients with severe achlorhydria. In many patients with gastric cancer, $H$. pylori infection is often difficult to detect even with multiple modalities (91). This is consistent with the observation that gastrin-deficient mice, which are achlorhydric, housed in a conventional facility develop bacterial overgrowth and chronic atrophic gastritis and progress to antral gastric cancer $(92,93)$. This pathological process is very similar to that which develops in $H$. felis-infected wild-type mice, again after long-standing achlorhydria and after Helicobacter spp. are almost undetectable (94). Evidence thus far indicates that $H$. pylori is more important as a trigger for the development of gastric atrophy and achlorhydria than as a promoter of advanced gastric cancer. However, it has become clear from studies in achlorhydric mice that bacteria other than Helicobacter spp. can induce atrophic gastritis (95).

Although one can hypothesize that the main role of $H$. pylori in gastric cancer is the induction of atrophic gastritis and achlorhydria, a role in gastric cancer progression for colonization with non-Helicobacter spp. has not been defined. Nevertheless, bacterial colonization of the achlorhydric stomach is likely to be important in gastric cancer progression, and one must consider that in $H$. pylori-infected patients, the beneficial effects of antibiotics might be as much from suppression of non-H. pylori organisms as it is from $H$. pylori eradication.

\section{Role of acid-suppressive drugs in the development of atrophy}

Given the probable role of achlorhydria in the development of bacterial overgrowth, what is the potential for powerful acid suppressants such as proton-pump inhibitors (PPIs) to accelerate the progression of gastric atrophy and gastric cancer? PPIs are one of the most commonly prescribed drugs and are generally perceived by most physicians as very effective and relatively safe. Indeed, in the largest mortality study, of 18,000 patients treated with omeprazole, there was no increased mortality that was not associated with preexisting illness (96). However, only $25 \%$ of the patients studied were on long-term therapy, and the length of follow-up might have been insufficient to detect an increase in gastric cancer. Long-term PPI therapy rarely has been associated with benign fundic gland polyps, but, to date, minimal changes in the number of enterochromaffin-like cells have been observed. However, the major concern that persists relates to the possible increase in atrophic gastritis in H. pylori-infected patients on long-term PPI therapy. As initially reported (97), the proportion of individuals with atrophic gastritis was greater among $H$. pylori-infected patients taking omeprazole to treat their reflux esophagitis than among $H$. pylori-infected patients receiving alternative treatment for their reflux esophagitis, and similar results have been noted by several other investigators (98-100). However, no increase in atrophic gastritis was observed in another study (101). Furthermore, although corpus atrophy is a probable precursor lesion to gastric cancer, no study to date has observed an increased risk for gastric cancer in PPI users.

It is generally accepted that PPI treatment can lead to a change in the distribution of $H$. pylori in the stomach from an antral to a predominantly corpus localization (102). Most studies have found an annual increase in corpus atrophy in $H$. pylori-infected patients on PPIs compared with those receiving no treatment $(99,100)$. One study has indicated that $H$. pylori-infected patients with the highest serum levels of gastrin before PPI therapy showed the most marked progression in gastric atrophy during PPI therapy (99). This notion that infection with $H$. pylori might accelerate atrophy in hypergastrinemic individuals has been supported by animal studies $(103,104)$. In mice overexpressing gastrin, the combination of hypergastrinemia and infection with Helicobacter spp. leads to increased parietal cell apoptosis and rapid progression to gastric atrophy and cancer (105). In addition, omeprazole treatment of $H$. felis-infected mice overexpressing gastrin resulted in progression to dysplasia (106). Given these findings, the strategy of testing and treating for infection with $H$. pylori before long-term PPI therapy is probably reasonable. Furthermore, the consequences of long-term achlorhydria and its influence on the human gastric microbiome are clearly worth investigating.

\section{Strategies for treating $H$. pylori infection in at-risk populations}

Decision analysis studies indicated that a strategy of screening and treating patients for infection with $H$. pylori would, in theory, be cost effective if $H$. pylori eradication could reduce the risk of developing gastric cancer risk by $30 \%$ or more (107). Initial studies from Japan involving $H$. pylori eradication in patients who had undergone partial gastrectomy for early gastric cancer suggested that eradication decreased the risk of tumor recurrence (108). Other studies indicate that antibiotic eradication of an $H$. pylori infection might reduce the risk of developing gastric cancer (109, $110)$. In one study (110), H. pylori eradication did not prevent cancer, although the trend was toward some diminution in the risk of developing disease. However, $H$. pylori eradication did reduce cancer risk in those patients who had no precancerous lesions when they entered the study (110). Although this study showed minimal benefit to patients with established atrophy, overall, studies of the 
effect of $H$. pylori eradication on gastric atrophy have been conflicting (111); some studies show improvements in atrophy with eradication $(112,113)$, whereas others show little or no improvement (114). The issue of reversibility of gastric atrophy has been examined in C57BL/6 mice infected with $H$. felis and then given 3 different antibiotics for 14 days 2, 6, or 12 months after infection (94). Antibiotic therapy 2 and 6 months after infection resulted in reversal of atrophy and restoration of normal gastric architecture, whereas bacterial eradication 1 year after infection resulted in some restoration of parietal cell mass and prevention of further progression of dysplasia in most animals (94). Although some questions have been raised regarding the worsening of gastroesophageal reflux after $H$. pylori eradication (115), most studies have not confirmed an increase in gastroesophageal reflux in patients whose H. pylori infection has been eradicated (116-119). Further studies are clearly needed to determine whether eliminating both Helicobacter and non-Helicobacter spp. from the gastric mucosa, through treatment with antibiotics, is of benefit, and to define the precise "point of no return" in human gastric cancer progression.

\section{Conclusion}

One of the major advances in our understanding of the host$H$. pylori interaction is how the evolutionary adaptation of the organism's virulence properties has allowed for both colonization and persistence in the stomach, which was historically considered a hostile milieu. Although bacterial virulence factors are important in determining disease outcome, it is the attempts of the immune system of the host to clear chronic infection with $H$. pylori that elicit an overly aggressive proinflammatory Th 1 cell response resulting in a robust gastritis that precedes a series of morphological changes that lead to cancer. Antibiotic intervention to eradicate $H$. pylori early in this pathological process might prevent progressively more severe disease. However, it is still unknown whether patients with advanced premalignant lesions can benefit from antibiotic eradication of $H$. pylori. Alternatively, mediators of chronic inflammation could also serve as potentially important therapeutic targets to prevent gastric cancer. For example, the setting of achlorhydria and sustained tissue damage in the stomach due to chronic inflammation might lead to cancer, in part by affecting myofibroblasts and altering the stem cell niche, thereby inducing stem cell recruitment. This model offers several avenues for potential prevention or treatment of cancer, through inhibition of inflammatory pathways and/or modulation of activated myofibroblasts. It has already been speculated that many chemoprevention agents already in existence may work in part through their effects on bone marrow-derived stem cells (120). Indeed, as has recently been stated, eradication of cancer stem cells will be required to improve outcome of treatment for selected cancers (121). Whether gastric cancer will be included in this category will require further studies.

\section{Acknowledgments}

This work was supported by NIH grants R01 AI37750, R01 AI50952, P01 CA26T31, and P30 ES02109 (to J.G. Fox) and grant R01 CA93405 (to T.C. Wang and J.G. Fox).

Address correspondence to: James G. Fox, Division of Comparative Medicine, Massachusetts Institute of Technology, 77 Massachusetts Avenue, Building 16-825C, Cambridge, Massachusetts 02139, USA. Phone: (617) 253-1735; Fax: (617) 252-1877; E-mail: jgfox@mit.edu.
1. Ries, L.A.G., et al. 2005. SEER Cancer Statistics Review, 1975-2002. National Cancer Institute. Bethesda, Maryland, USA. http://seer.cancer.gov/ csr/1975_2002/.

2. Parkin, D.M., Pisani, P., and Ferlay, J. 1999. Global cancer statistics. CA Cancer J. Clin. 49:33-64.

3. Lauren, P. 1965. The two histological main types of gastric carcinoma: diffuse and so-called intestinaltype carcinoma. An attempt at a histo-clinical classification. Acta Pathol. Microbiol. Scand. 64:31-49.

4. Cuello, C., et al. 1979. Histopathology of gastric dysplasias: correlations with gastric juice chemistry. Am. J. Surg. Pathol. 3:491-500.

5. Philip, M., Rowley, D.A., and Schreiber, H. 2004. Inflammation as a tumor promoter in cancer induction. Semin. Cancer Biol. 14:433-439.

6. Gilmour, J. 1958. Clinical aspects of carcinoma of stomach in diagnosis based upon the study of 146 cases. Br. Med. J. 5073:745-748.

7. Gilmour, J. 1961. The surgical aspects of gastritis and its role in gastric pathology. Br. J. Surg. 49:278-288.

8. Siurala, M., Isokoski, M., Varis, K., and Kekki, M. 1968. Prevalence of gastritis in a rural population. Bioptic study of subjects selected at random. Scand. J. Gastroenterol. 3:211-223.

9. Correa, P. 1980. The epidemiology and pathogenesis of chronic gastritis: three etiologic entities. Front. Gastrointest. Res. 6:98-108.

10. Correa, P. 1985. Clinical implications of recent developments in gastric cancer pathology and epidemiology. Semin. Oncol. 12:2-10.

11. Correa, P. 1983. Chronic atrophic gastritis as a precursor of cancer. In Precancerous lesions of the gastrointestinal tract. P. Sherlock, B. Morson, L. Barbara, and U. Veronesi, editors. Raven Press. New York, New York, USA. 145-153.

12. Bonne, C., et al. 1938. Morphology of the stomach and gastric secretion in Malays and Chinese and the different incidence of gastric ulcer and cancer in these races. Am. J. Cancer. 33:265-279.

13. Siurala, M., Varis, K., and Wiljasalo, M. 1966. Studies of patients with atrophic gastritis: a 10-15-year follow-up. Scand. J. Gastroenterol. 1:40-48.

14. Fox, J.G., and Wang, T.C. 2001. Helicobacter pylorinot a good bug after all! N. Engl. J. Med. 345:829-832.

15. Correa, P. 1995. Helicobacter pylori and gastric carcinogenesis. Am. J. Surg. Pathol. 19(Suppl. 1):S37-S43.

16. Genta, R.M. 1997. Helicobacter pylori, inflammation, mucosal damage, and apoptosis: pathogenesis and definition of gastric atrophy. Gastroenterology. 113(Suppl. 6):S51-S55.

17. El-Zimaity, H.M., et al. 2002. Patterns of gastric atrophy in intestinal type gastric carcinoma. Cancer. 94:1428-1436.

18. Schmidt, P.H., et al. 1999. Identification of a metaplastic cell lineage associated with human gastric adenocarcinoma. Lab. Invest. 79:639-646.

19. Halldorsdottir, A.M., et al. 2003. Spasmolytic polypeptide-expressing metaplasia (SPEM) associated with gastric cancer in Iceland. Dig. Dis. Sci. 48:431-441.

20. Fox, J.G., et al. 1996. Hypertrophic gastropathy in Helicobacter felis-infected wild-type C57BL/6 mice and p53 hemizygous transgenic mice. Gastroenterology. 110:155-166.

21. Wang, T.C., et al. 1998. Mice lacking secretory phospholipase A2 show altered apoptosis and differentiation with Helicobacter felis infection. Gastroenterology. 114:675-689.

22. Li, Q., Karam, S.M., and Gordon, J.I. 1996. Diphtheria toxin-mediated ablation of parietal cells in the stomach of transgenic mice. J. Biol. Chem. 271:3671-3676.

23. Van den Brink, G.R., et al. 2001. Sonic hedgehog regulates gastric gland morphogenesis in man and mouse. Gastroenterology. 121:317-328.

24. Shiotani, A., et al. 2005. Evidence that loss of sonic hedgehog is an indicator of Helicobacter pyloriinduced atrophic gastritis progressing to gastric cancer. Am. J. Gastroenterol. 100:581-587.

25. Ramalho-Santos, M., Melton, D.A., and McMahon, A.P. 2000. Hedgehog signals regulate multiple aspects of gastrointestinal development. Development. 127:2763-2772.

26. Goldenring, J.R., et al. 2000. Reversible druginduced oxyntic atrophy in rats. Gastroenterology. 118:1080-1093.

27. Nomura, S., et al. 2005. Alterations in gastric mucosal lineages induced by acute oxyntic atrophy in wild-type and gastrin-deficient mice. Am. J. Physiol. Gastrointest. Liver Physiol. 288:G362-G375.

28. Houghton, J., et al. 2004. Gastric cancer originating from bone marrow-derived cells. Science. 306:1568-1571.

29. Taylor, D., and Parsonnet, J. 1995. Infections of the gastrointestinal tract. In Infections of the gastrointestinal tract. M.J. Blaser, P. Smith, J. Ravdin, H. Greenberg, and R. Guerrant, editors. Raven Press. New York, New York, USA. 551-563.

30. Hansson, L.E., et al. 1996. The risk of stomach cancer in patients with gastric or duodenal ulcer disease. N. Engl. J. Med. 335:242-249.

31. Oh, J.D., Karam, S.M., and Gordon, J.I. 2005. Intracellular Helicobacter pylori in gastric epithelial progenitors. Proc. Natl. Acad. Sci. U. S. A. 102:5186-5191.

32. Wilkinson, S.M., Uhl, J.R., Kline, B.C., and Cockerill, F.R., 3rd. 1998. Assessment of invasion frequencies of cultured HEp-2 cells by clinical isolates of Helicobacter pylori using an acridine orange assay. J. Clin. Pathol. 51:127-133.

33. Semino-Mora, C., et al. 2003. Intracellular and interstitial expression of Helicobacter pylori virulence 
genes in gastric precancerous intestinal metaplasia and adenocarcinoma. J. Infect. Dis. 187:1165-1177.

34. Ilver, D., et al. 1998. Helicobacter pylori adhesin binding fucosylated histo-blood group antigens revealed by retagging. Science. 279:373-377.

35. Backstrom, A., et al. 2004. Metastability of Helicobacter pylori bab adhesin genes and dynamics in Lewis b antigen binding. Proc. Natl. Acad. Sci. U. S. A. 101:16923-16928.

36. Gerhard, M., et al. 1999. Clinical relevance of the Helicobacter pylori gene for blood-group antigen-binding adhesin. Proc. Natl. Acad. Sci. U. S. A. 96:12778-12783

37. Hansson, L.E., et al. 1993. Helicobacter pylori infection: independent risk indicator of gastric adenocarcinoma. Gastroenterology. 105:1098-1103.

38. Mahdavi, J., et al. 2002. Helicobacter pylori SabA adhesin in persistent infection and chronic inflammation. Science. 297:573-578.

39. Linden, S., et al. 2002. Strain- and blood groupdependent binding of Helicobacter pylori to human gastric MUC5AC glycoforms. Gastroenterology. 123:1923-1930.

40. Clyne, M., et al. 2004. Helicobacter pylori interacts with the human single-domain trefoil protein TFF1. Proc. Natl. Acad. Sci. U. S. A. 101:7409-7414.

41. Peek, R.M., Jr., and Crabtree, J.E. 2006. Helicobacter infection and gastric neoplasia. J. Pathol. 208:233-248.

42. Parsonnet, J., Friedman, G.D., Orentreich, N., and Vogelman, H. 1997. Risk for gastric cancer in people with CagA positive or CagA negative Helicobacter pylori infection. Gut. 40:297-301.

43. Enroth, H., et al. 2000. Helicobacter pylori strain types and risk of gastric cancer: a case-control study. Cancer Epidemiol. Biomarkers Prev. 9:981-985.

44. Shibata, A., et al. 2002. CagA status of Helicobacter pylori infection and $\mathrm{p} 53$ gene mutations in gastric adenocarcinoma. Carcinogenesis. 23:419-424.

45. Jenks, P.J., et al. 2003. Long-term infection with Helicobacter felis and inactivation of the tumour suppressor gene p53 cumulatively enhance the gastric mutation frequency in Big Blue transgenic mice. J. Pathol. 201:596-602

46. Higashi, H., et al. 2002. SHP-2 tyrosine phosphatase as an intracellular target of Helicobacter pylori CagA protein. Science. 295:683-686.

47. Tsutsumi, R., et al. 2003. Attenuation of Helicobacter pylori CagA $x$ SHP-2 signaling by interaction between CagA and C-terminal Src kinase. J. Biol. Chem. 278:3664-3670.

48. Higashi, H., et al. 2002. Biological activity of the Helicobacter pylori virulence factor CagA is determined by variation in the tyrosine phosphorylation sites. Proc. Natl. Acad. Sci. U. S. A. 99:14428-14433.

49. Crabtree, J.E., et al. 1995. Induction of interleukin-8 secretion from gastric epithelial cells by a cagA negative isogenic mutant of Helicobacter pylori. J. Clin. Pathol. 48:967-969.

50. Censini, S., et al. 1996. cag, a pathogenicity island of Helicobacter pylori, encodes type I-specific and disease-associated virulence factors. Proc. Natl. Acad. Sci. U. S. A. 93:14648-14653.

51. Segal, E.D., et al. 1997. Induction of host signal transduction pathways by Helicobacter pylori. Proc. Natl. Acad. Sci. U. S. A. 94:7595-7599.

52. Viala, J., et al. 2004. Nod1 responds to peptidoglycan delivered by the Helicobacter pylori cag pathogenicity island. Nat. Immunol. 5:1166-1174.

53. Leunk, R.D., et al. 1988. Cytotoxic activity in brothculture filtrates of Campylobacter pylori. J. Med. Microbiol. 26:93-99.

54. Harris, P.R., et al. 1996. Helicobacter pylori cytotoxin induces vacuolation of primary human mucosal epithelial cells. Infect. Immun. 64:4867-4871.

55. Smoot, D.T., et al. 1996. Effects of Helicobacter pylori vacuolating cytotoxin on primary cultures of human gastric epithelial cells. Gut. 39:795-799.
56. Atherton, J.C., et al. 1995. Mosaicism in vacuolating cytotoxin alleles of Helicobacter pylori. Association of specific vacA types with cytotoxin production and peptic ulceration. J. Biol. Chem. 270:17771-17777.

57. Garza-Gonzalez, E., et al. 2004. Association of gastric cancer, HLA-DQA1, and infection with Helicobacter pylori $\mathrm{CagA}+$ and $\mathrm{VacA}+$ in a Mexican population. J. Gastroenterol. 39:1138-1142.

58. Figueiredo, C., et al. 2002. Helicobacter pylori and interleukin 1 genotyping: an opportunity to identify high-risk individuals for gastric carcinoma. J. Natl. Cancer Inst. 94:1680-1687.

59. Bravo, L.E., van Doom, L.J., Realpe, J.L., and Correa, P. 2002. Virulence-associated genotypes of Helicobacter pylori: do they explain the African enigma? Am. J. Gastroenterol. 97:2839-2842.

60. Perez-Perez, G.I., Garza-Gonzalez, E., Portal, C., and Olivares, A.Z. 2005. Role of cytokine polymorphisms in the risk of distal gastric cancer development. Can cer Epidemiol. Biomarkers Prev. 14:1869-1873.

61. Gebert, B., et al. 2003. Helicobacter pylori vacuolating cytotoxin inhibits T lymphocyte activation. Science. 301:1099-1102.

62. Koh, T., and Wang, T. 2002. Tumors of the stomach. In Sleisenger e' Fordtran's gastrointestinal and liver disease: pathophysiology, diagnosis, management. M. Feldman, L. Friedman, and M. Sleisenger, editors. W.B. Saunders Co. Philadelphia, Pennsylvania, USA. 829-855.

63. El-Omar, E.M., et al. 2000. Interleukin-1 polymorphisms associated with increased risk of gastric cancer. Nature. 404:398-402.

64. Machado, J.C., et al. 2001. Interleukin 1B and interleukin 1RN polymorphisms are associated with increased risk of gastric carcinoma. Gastroenterology. 121:823-829.

65. Furuta, T., Shirai, N., and Sugimoto, M. 2004. Controversy in polymorphisms of interleukin-1beta in gastric cancer risks. J. Gastroenterol. 39:501-503.

66. Palli, D., et al. 2005. Interleukin-1 gene polymorphisms and gastric cancer risk in a high-risk Italian population. Am. J. Gastroenterol. 100:1941-1948.

67. El-Omar, E.M., et al. 2003. Increased risk of noncardia gastric cancer associated with proinflammatory cytokine gene polymorphisms. Gastroenterology. 124:1193-1201.

68. Lee, W P et al 2005. The -251T allele of the interleukin-8 promoter is associated with increased risk of gastric carcinoma featuring diffuse-type histopathology in Chinese population. Clin. Cancer Res. 11:6431-6441

69. Taguchi, A., et al. 2005. Interleukin-8 promoter polymorphism increases the risk of atrophic gastritis and gastric cancer in Japan. Cancer Epidemiol. Biomarkers Prev. 14:2487-2493.

70. Roth, K.A., Kapadia, S.B., Martin, S.M., and Lorenz, R.G. 1999. Cellular immune responses are essential for the development of Helicobacter felis-associated gastric pathology. J. Immunol. 163:1490-1497.

71. Eaton, K.A., Ringler, S.R., and Danon, S.J. 1999. Murine splenocytes induce severe gastritis and delayed-type hypersensitivity and suppress bacterial colonization in Helicobacter pylori-infected SCID mice. Infect. Immun. 67:4594-4602.

72. Eaton, K.A., Mefford, M., and Thevenot, T. 2001. The role of $\mathrm{T}$ cell subsets and cytokines in the pathogenesis of Helicobacter pylori gastritis in mice. J. Immunol. 166:7456-7461.

73. McCracken, V.J., Martin, S.M., and Lorenz, R.G. 2005. The Helicobacter felis model of adoptive transfer gastritis. Immunol. Res. 33:183-194.

74. Fox, J.G., et al. 2000. Concurrent enteric helminth infection modulates inflammation and gastric immune responses and reduces helicobacterinduced gastric atrophy. Nat. Med. 6:536-542.

75. Smythies, L.E., et al. 2000. Helicobacter pyloriinduced mucosal inflammation is Th1 mediated and exacerbated in IL-4, but not IFN-gamma, gene- deficient mice. J. Immunol. 165:1022-1029.

76. Kang, W., Rathinavelu, S., Samuelson, L.C., and Merchant, J.L. 2005. Interferon gamma induction of gastric mucous neck cell hypertrophy. Lab. Invest. 85:702-715.

77. Zavros, Y., et al. 2003. Treatment of Helicobacter gastritis with IL-4 requires somatostatin. Proc. Natl. Acad. Sci. U. S. A. 100:12944-12949.

78. Mitchell, H.M., et al. 2002. Major differences in the IgG subclass response to Helicobacter pylori in the first and third worlds. Scand. J. Gastroenterol. 37:517-522.

79. Whary, M.T., et al. 2005. Intestinal helminthiasis in Colombian children promotes a Th2 response to Helicobacter pylori: possible implications for gastric carcinogenesis. Cancer Epidemiol. Biomarkers Prev. 14:1464-1469.

80. Elshal, M.F., et al. 2004. Role of concurrent S. mansoni infection in $H$. pylori-associated gastritis: a flow cytometric DNA-analysis and oxyradicals correlations. Clin. Chim. Acta. 346:191-198.

81. Du, Y., et al. 2006. Helicobacter pylori and Schistosoma japonicum co-infection in a Chinese population: helminth infection alters humoral responses to H. pylori and serum pepsinogen I/II ratio. Microbes Infect. 8:52-60.

82. Brittan, M., et al. 2002. Bone marrow derivation of pericryptal myofibroblasts in the mouse and human small intestine and colon. Gut. 50:752-757.

83. Forbes, S.J., et al. 2004. A significant proportion of myofibroblasts are of bone marrow origin in human liver fibrosis. Gastroenterology. 126:955-963.

84. Brittan, M., et al. 2005. A regenerative role for bone marrow following experimental colitis: contribution to neovasculogenesis and myofibroblasts. Gastroenterology. 128:1984-1995.

85. Houghton, J., Morozov, A., Smirnova, I., and Wang, T.C. 2006. Stem cells and cancer. Semin. Cancer Biol. doi:10.1016/j.semcancer.2006.04.003.

86. Wang, X., et al. 2003. Cell fusion is the principal source of bone-marrow-derived hepatocytes. Nature. 422:897-901.

87. Rizvi, A.Z., et al. 2006. Bone marrow-derived cells fuse with normal and transformed intestinal stem cells. Proc. Natl. Acad. Sci. U. S. A. 103:6321-6325.

88. Camargo, F.D., Chambers, S.M., and Goodell, M.A. 2004. Stem cell plasticity: from transdifferentiation to macrophage fusion. Cell Prolif. 37:55-65.

89. Orimo, A., et al. 2005. Stromal fibroblasts present in invasive human breast carcinomas promote tumor growth and angiogenesis through elevated SDF-1/CXCL12 secretion. Cell. 121:335-348.

90. Williams, C., and McColl, K.E. 2006. Review article: proton pump inhibitors and bacterial overgrowth. Aliment. Pharmacol. Ther. 23:3-10.

91. Ekstrom, A.M., et al. 2001. Helicobacter pylori in gastric cancer established by CagA immunoblot as a marker of past infection. Gastroenterology. 121:784-791.

92. Zavros, Y., et al. 2005. Chronic gastritis in the hypochlorhydric gastrin-deficient mouse progresses to adenocarcinoma. Oncogene. 24:2354-2366.

93. Zavros, Y., et al. 2002. Genetic or chemical hypochlorhydria is associated with inflammation that modulates parietal and G-cell populations in mice. Gastroenterology. 122:119-133.

94. Cai, X., et al. 2005. Helicobacter felis eradication restores normal architecture and inhibits gastric cancer progression in C57BL/6 mice. Gastroenterology. 128:1937-1952.

95. Rathinavelu, S., Zavros, Y., and Merchant, J.L. 2003. Acinetobacter lwoffii infection and gastritis. Microbes Infect. 5:651-657.

96. Bateman, D.N., et al. 2003. Mortality study of 18,000 patients treated with omeprazole. Gut. 52:942-946.

97. Kuipers, E.J., et al. 1996. Atrophic gastritis and Helicobacter pylori infection in patients with reflux esophagitis treated with omeprazole or fundoplica- 
tion. N. Engl. J. Med. 334:1018-1022.

98. Berstad, A.E., et al. 1997. Helicobacter pylori gastritis and epithelial cell proliferation in patients with reflux oesophagitis after treatment with lansoprazole. Gut. 41:740-747.

99. Eissele, R., et al. 1997. Gastric mucosa during treatment with lansoprazole: Helicobacter pylori is a risk factor for argyrophil cell hyperplasia. Gastroenterology. 112:707-717.

100.Klinkenberg-Knol, E.C., et al. 2000. Long-term omeprazole treatment in resistant gastroesophageal reflux disease: efficacy, safety, and influence on gastric mucosa. Gastroenterology. 118:661-669.

101.Lundell, L. 2000. Lack of effect of acid suppression therapy on gastric atrophy. Gastroenterology. 118:453.

102.Larkin, C.J., et al. 2000. Distribution of atrophy in Helicobacter pylori-infected subjects taking proton pump inhibitors. Scand. J. Gastroenterol. 35:578-582.

103. Wang, T.C., et al. 2000. Synergistic interaction between hypergastrinemia and Helicobacter infection in a mouse model of gastric cancer. Gastroenterology. 118:36-47.

104. Fox, J.G., et al. 2003. Host and microbial constituents influence Helicobacter pylori-induced cancer in a murine model of hypergastrinemia. Gastroenterology. 124:1879-1890.

105.Cui, G., et al. 2006. Gastrin-induced apoptosis contributes to carcinogenesis in the stomach. Lab. Invest. 86:1037-1051.

106. Takaishi, S., et al. 2005. Synergistic inhibitory effects of gastrin and histamine receptor antagonists on Helicobacter-induced gastric cancer. Gastroenterology. 128:1965-1983.

107. Parsonnet, J., Harris, R.A., Hack, H.M., and Owens,
D.K. 1996. Modelling cost-effectiveness of Helicobacter pylori screening to prevent gastric cancer: a mandate for clinical trials. Lancet. 348:150-154.

108.Uemura, N., et al. 1997. Effect of Helicobacter pylori eradication on subsequent development of cancer after endoscopic resection of early gastric cancer. Cancer Epidemiol. Biomarkers Prev. 6:639-642.

109.Uemura, N., et al. 2001. Helicobacter pylori infection and the development of gastric cancer. N. Engl. J. Med. 345:784-789.

110.Wong, B.C., et al. 2004. Helicobacter pylori eradication to prevent gastric cancer in a high-risk region of China: a randomized controlled trial. JAMA. 291:187-194.

111.Zivny, J., et al. 2003. Role of therapy or monitoring in preventing progression to gastric cancer. J. Clin. Gastroenterol. 36(Suppl. 5):S50-S60; discussion S61-S62.

112.Zhou, L., et al. 2003. A five-year follow-up study on the pathological changes of gastric mucosa after H. pylori eradication. Chin. Med. J. (Engl.) 116:11-14.

113.Ito, M., et al. 2002. Helicobacter pylori eradication therapy improves atrophic gastritis and intestinal metaplasia: a 5-year prospective study of patients with atrophic gastritis. Aliment. Pharmacol. Ther. 16:1449-1456.

114.Ruiz, B., et al. 2001. Morphometric evaluation of gastric antral atrophy: improvement after cure of Helicobacter pylori infection. Am. J. Gastroenterol. 96:3281-3287.

115.Labenz, J., et al. 1997. Curing Helicobacter pylori infection in patients with duodenal ulcer may provoke reflux esophagitis. Gastroenterology. 112:1442-1447.

116. Moayyedi, P., et al. 2001. Helicobacter pylori eradication does not exacerbate reflux symptoms in gastroesophageal reflux disease. Gastroenterology. 121:1120-1126.

117. Schwizer, W., et al. 2001. Helicobacter pylori and symptomatic relapse of gastro-oesophageal reflux disease: a randomised controlled trial. Lancet. 357:1738-1742.

118. Kuipers, E.J., et al. 2004. Cure of Helicobacter pylori infection in patients with reflux oesophagitis treated with long term omeprazole reverses gastritis without exacerbation of reflux disease: results of a randomised controlled trial. Gut. 53:12-20.

119.Vaira, D., et al. 2003. Effect of Helicobacter pylori eradication on development of dyspeptic and reflux disease in healthy asymptomatic subjects. Gut. 52:1543-1547.

120. Wang, T.C. 2006. Stem cells and cancer prevention. Cancer Prevention. Issue 7. http://www.nypcancerprevention.com/issue/7/pro/iss/issues-insights.shtml.

121.Jordan, C.T., Guzman, M.L., and Noble, M. 2006. Cancer stem cells. N. Engl. J. Med. 355:1253-1261.

122.Leeson, C., Leeson, T., and Paparo, A. 1985. Textbook of histology. W.B. Saunders Co. Philadelphia, Pennsylvania, USA. 597 pp.

123. Correa, P. 1991. Is gastric carcinoma an infectious disease? N. Engl. J. Med. 325:1170-1171.

124.Rogers, A.B., and Fox, J.G. 2004. Inflammation and cancer. I. Rodent models of infectious gastrointestinal and liver cancer. Am. J. Physiol. Gastrointest. Liver Physiol. 286:G361-G366.

125. Rogers, A.B., et al. 2005. Helicobacter pylori but not high salt induces gastric intraepithelial neoplasia in B6129 mice. Cancer Res. 65:10709-10715.

126.Fox, J.G., et al. 2003. Helicobacter pylori-associated gastric cancer in INS-GAS mice is gender specific. Cancer Res. 63:942-950. 